\title{
Physical Function and Health Related Quality of Life Before and 18 Months after Bariatric Surgery
}

\author{
Malin Wiklund ${ }^{1,2, *}$ and Monika F. Olsén ${ }^{1,2,3}$ \\ ${ }^{I}$ Department of Physiotherapy and Occupational Therapy, Sahlgrenska University Hospital, Gothenburg, Sweden \\ ${ }^{2}$ Institute of Clinical Sciences, Department of Gastrosurgical Research and Education, Sahlgrenska Academy, \\ University of Gothenburg, Gothenburg, Sweden \\ ${ }^{3}$ Institute of Neuroscience and Physiology, Department of Physiotherapy, Sahlgrenska Academy, University of \\ Gothenburg, Gothenburg, Sweden
}

\begin{abstract}
Introduction: The mobility disability experienced by people with obesity is well known and has been found to be associated with reduced health related quality of life (HRQoL) compared to people without obesity. Research is lacking related to the patients experiences that how their capacity to perform various daily physical activities and HRQoL are affected by weight loss following bariatric surgery.

Aim: To evaluate patients' experiences of their HRQoL and physical function before and 18 months after laparoscopic Roux-en-Y Gastric Bypass surgery (LRYGB).

Method: A series of 70 patients filled in one HRQoL questionnaire, the EQ-5D (including EQ VAS and EQ-5D descriptive system) and two self-assessment questionnaires that evaluate disability by assessing activity and participation limitations, the Disability Rating Index (DRI) and a questionnaire with five disease-specific questions.

Results: All activities, in both DRI as well as the five disease-specific questions were experienced as significantly less difficult to perform postoperatively than preoperatively $(\mathrm{p}<0.05)$. In this study, the median ( $\min , \max )$ EQ VAS score was $60(20,100) \mathrm{mm}$ preoperatively and $80(20,100) \mathrm{mm}$ postoperatively $(100=$ best imaginable health; $0=$ worst imaginable health). The difference between pre- and postoperative EQ VAS was significant $(\mathrm{p}<0.001)$.
\end{abstract}

Conclusion: Both the HRQoL and the self experienced ability to perform various daily physical activities increased significantly 18 months after LRYGB compared to preoperatively.

Keywords: Bariatric surgery, gastric by-pass surgery, disability, obesity, physical function, quality of life.

\section{INTRODUCTION}

Obesity causes substantial health problems and the impact on a decrease in mobility and disability is well known. The mobility disability experienced by people with obesity has been found to be associated with reduced health related quality of life (HRQoL) as compared to people without obesity [1].

Bariatric surgery is associated with greater long-term weight loss than conventional treatment, as well as a lower risk of disease and decreased overall mortality [2, 3]. Bariatric surgery has also been found to result in substantial, long-term improvements in HRQoL [4], but research is lacking concerning patients experiences that how their capacity to perform various daily physical activities is affected by the surgery-induced weight loss.

*Address correspondence to this author at the Department of Physiotherapy, Sahlgrenska University Hospital, 41345 Gothenburg, Sweden;

Tel: +46313429321; Fax: +46313424341;

E-mail: malin.wiklund@ @gregion.se
The Disability Rating Index (DRI) is a patient-reported outcome measure, developed by Salén et al. in 1994 [5]. It evaluates a patient's disability (physical function) by assessing activity and participation limitations. DRI is constructed as a self-administered form and covers 12 items on physical function. Patients indicate their presumed ability to perform various daily physical activities (from dressing and sitting to participating in sports) on a visual analogue scale (VAS scale) [5]. There is currently no trial that evaluates the degree of physical function measured by DRI before and after bariatric surgery,

Even though DRI covers important daily activities, people with obesity have specific limitations due to the fat and extra tissue. Examples of such activities are bending down to tie shoes and rising from a chair. Studies concerning the degree of limitation in such activities before and after bariatric surgery are lacking.

The aim of this study was therefore to evaluate patients' experiences of their HRQoL and physical function before and 18 months after laparoscopic Roux-en-Y Gastric Bypass surgery (LRYGB). 


\section{METHOD}

A series of 70 patients filled in one HRQoL questionnaire and two self-assessment questionnaires concerning their physical capacity/function before and 18 months after LRYGB, performed at a university hospital in Sweden. All patients were included after written and verbal information and written consent. The Regional Ethical Review Board of Gothenburg, Sweden, approved the study, and it was registered in a national register for clinical trials before the start of the study.

Physical function was assessed by the Disability Rating Index (DRI). This is a patient-reported outcome measure that was developed by Salén et al. in 1994 [5] and evaluates a patient's disability by assessing activity and participation limitations. The DRI questionnaire is self-administered and quick to complete $(<5 \mathrm{~min})$. It is a single scale comprising 12 items, and patients indicate their presumed ability (capacity) to perform various daily physical activities on a $100-\mathrm{mm}$ visual analogue scale (VAS scale) where zero corresponds to "manage without difficulty" and 100 millimeters is equivalent to "cannot manage at all". The DRI items can be analyzed separately and/or as a total mean index score, the DRI Index. The instrument has been used in the clinical setting since 1980 and was primarily developed for patients with low back pain. DRI discriminates well between different diagnostic categories and shows sensitivity for small age and gender differences. It has shown high reliability with test-retest correlations of $0.83-0.95$ and intraand inter-rate reproducibility of 0.98 and 0.99 , respectively [5]. The DRI provides a global, rather than a localized or joint-/function-specific, outcome assessment and has been found to be robust, reliable and sensitive. It has been endorsed for use across a wide range of clinical and research settings, including randomized controlled trials and routine patient follow-up [6].

In addition, the patients assessed five disease-specific functions: "rise from a chair without arm support", "tying shoes", "bend over and pick up something from the floor", "wipe your butt after using the toilet" and "vacuum cleaning". These questions were selected by two specialist physiotherapists with many years of experience of working with this patient population. These questions were answered in the same way as the DRI, i.e. on a 100-mm VAS scale.

The HRQoL questionnaire, EQ-5D, is a standardized non-disease-specific instrument for describing and evaluating HRQoL and is designed to monitor the health status of patient groups at different points in time. In the EQ VAS, patients rate their own overall health status on a vertical VAS $(100=$ best imaginable health; $0=$ worst imaginable health). With the EQ-5D descriptive system, patients classify their own health status in five dimensions including mobility, self-care, usual activities, pain/discomfort, anxiety/depression, on three levels of severity, no problems; moderate problems; severe problems [7]. EQ-5D health states, defined by the EQ-5D descriptive system, may be converted into a single summary index (the EQ-5D index) by applying a formula that essentially attaches values (also called weights) to each of the levels in each dimension (i.e. if the patient scores 11111 in the five items, this gives a value of 0.9694). Value sets have been derived for EQ-5D in several countries, including Sweden [8].

\section{STATISTICAL ANALYSES}

Statistical analyses were carried out using SPSS version 22.0 , and the statistical significance level was set at $\mathrm{p}<0.05$ (two-sided test). The Wilcoxon signed rank test was used to evaluate differences between pre- and postoperative measurements. The Spearman correlation coefficient $\left(r_{s}\right)$ was used to assess the relationship between the differences in weight or BMI and the items in DRI and the five additional questions. The Spearman correlation coefficient $\left(\mathrm{r}_{\mathrm{s}}\right)$ was also used to assess the relationship between EQ-5D and the DRI index. Correlation was defined in this study as: little, if any $\left(r_{\mathrm{s}}<0.25\right)$, low $\left(r_{\mathrm{s}}=0.26-0.49\right)$, moderate $\left(\mathrm{r}_{\mathrm{s}}=0.50-0.69\right)$, high $\left(r_{s}=0.70-0.89\right)$ and very high $\left(r_{s}=0.9-1.00\right)$ [9].

\section{RESULTS}

The 70 patients ( 41 women and 29 men) had a mean (SD) age of 47(12) years and a mean height of $1.73(0.1) \mathrm{m}$. The patients' weight changed from 133.7 (24.5) $\mathrm{kg}$ preoperatively to 93.1 (18.4) $\mathrm{kg}$ postoperatively giving a change in mean body mass index (BMI) of $44.7(5.8) \mathrm{kg} / \mathrm{m}^{2}$ preoperatively to $31.2(5.2) \mathrm{kg} / \mathrm{m}^{2}$ postoperatively.

Fig. (1) shows the median in $\mathrm{mm}$ for each activity, assessed by the DRI, as well as the total mean index score (the DRI Index). The total mean index score was $30.4 \mathrm{~mm}$ (min $1.9 \mathrm{~mm}$ and $\max 79.1$ ) before surgery. Corresponding values 18 months after surgery were $14.2 \mathrm{~mm}(\min 0 \mathrm{~mm}$ and $\max 82.5 \mathrm{~mm}) \quad(\mathrm{p}<0.001)$. All activities were experienced as being less difficult to perform postoperatively than preoperatively. "Run" was the activity that was the most difficult to perform; the preoperative median was $74.4 \mathrm{~mm}$ and the postoperative median was $40.7 \mathrm{~mm}$. There was a significant difference in pre-and postoperative values in all activities $(\mathrm{p}<0.001)$ for all items except in "sitting for a longer time" with a p-value of 0.026 .

Low correlations were found between preoperative weight or BMI and the separate activities assessed in DRI ( $\mathrm{r}_{\mathrm{s}}$ $<0.37)$, the total mean index score $\left(r_{s}=0.27\right)$ and the additional five separate questions $\left(r_{s}<0.32\right)$. The same pattern was seen 18 months postoperatively with overall low correlations $\left(\mathrm{r}_{\mathrm{s}}<0.23\right)$. The correlation coefficients between difference in weight or BMI (preoperative-postoperative) to difference in DRI were $\mathrm{r}_{\mathrm{s}}=0.273, \mathrm{p}<0.001$ (weight), and $\mathrm{r}_{\mathrm{s}}=0.273, \mathrm{p}=0.022$ (BMI).

Fig. (2) illustrates the median in millimeters for the five activities with known difficulties in the patient group preand postoperatively. All activities were experienced as being more difficult to perform preoperatively than postoperatively; "bend over and pick up something from the floor" was the activity that was most difficult to perform preoperatively: median $29.1 \mathrm{~mm}$. This activity significantly improved 18 months postoperatively to, in median, $3.7 \mathrm{~mm}$. There was a significant difference in pre- and postoperative values in all activities ( $\mathrm{p}<0.001)$. 


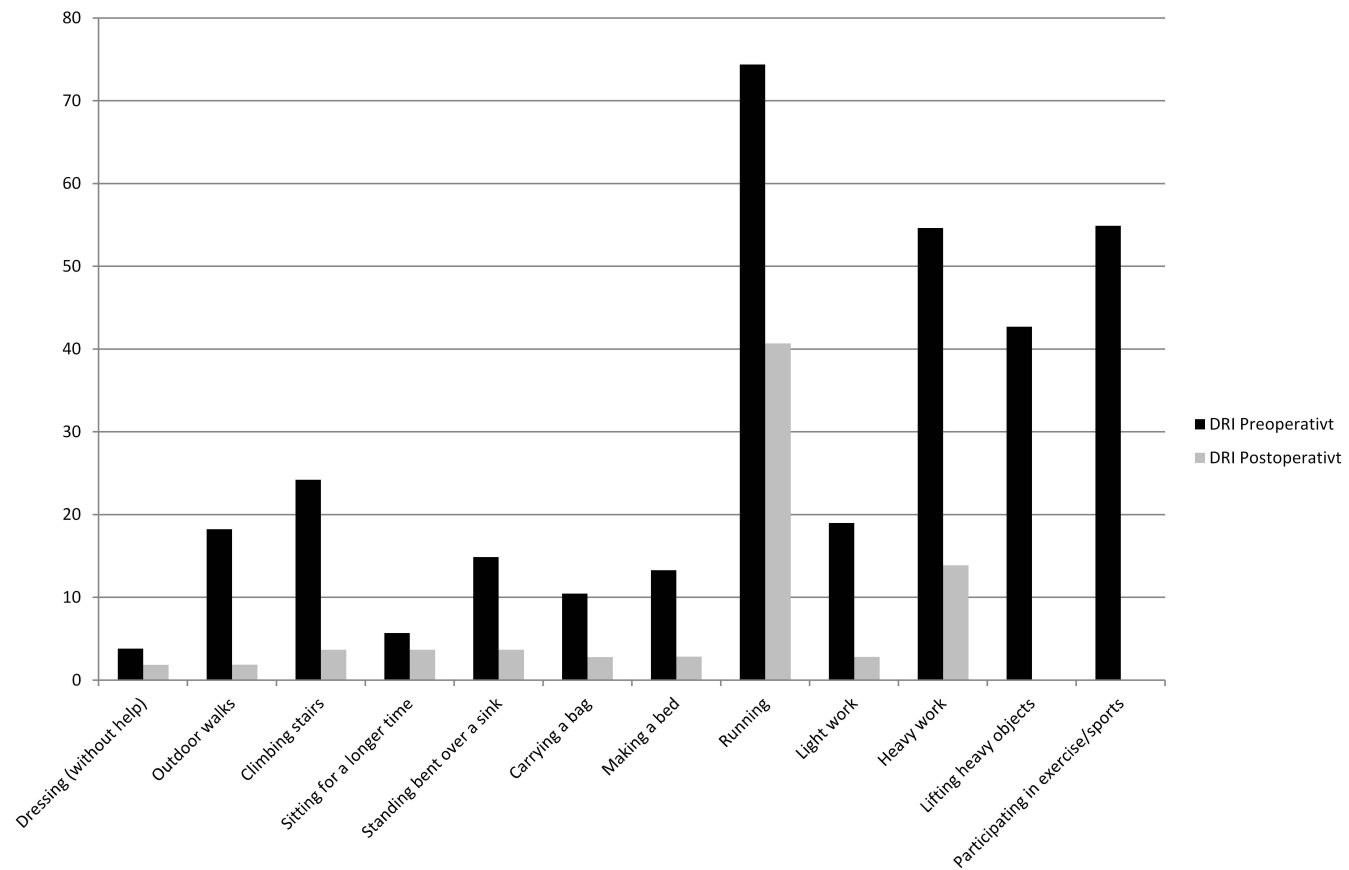

Fig. (1). The median of the 12 items of DRI (in millimeters) and the total mean index score for the 70 patients preoperatively and 18 months postoperatively.

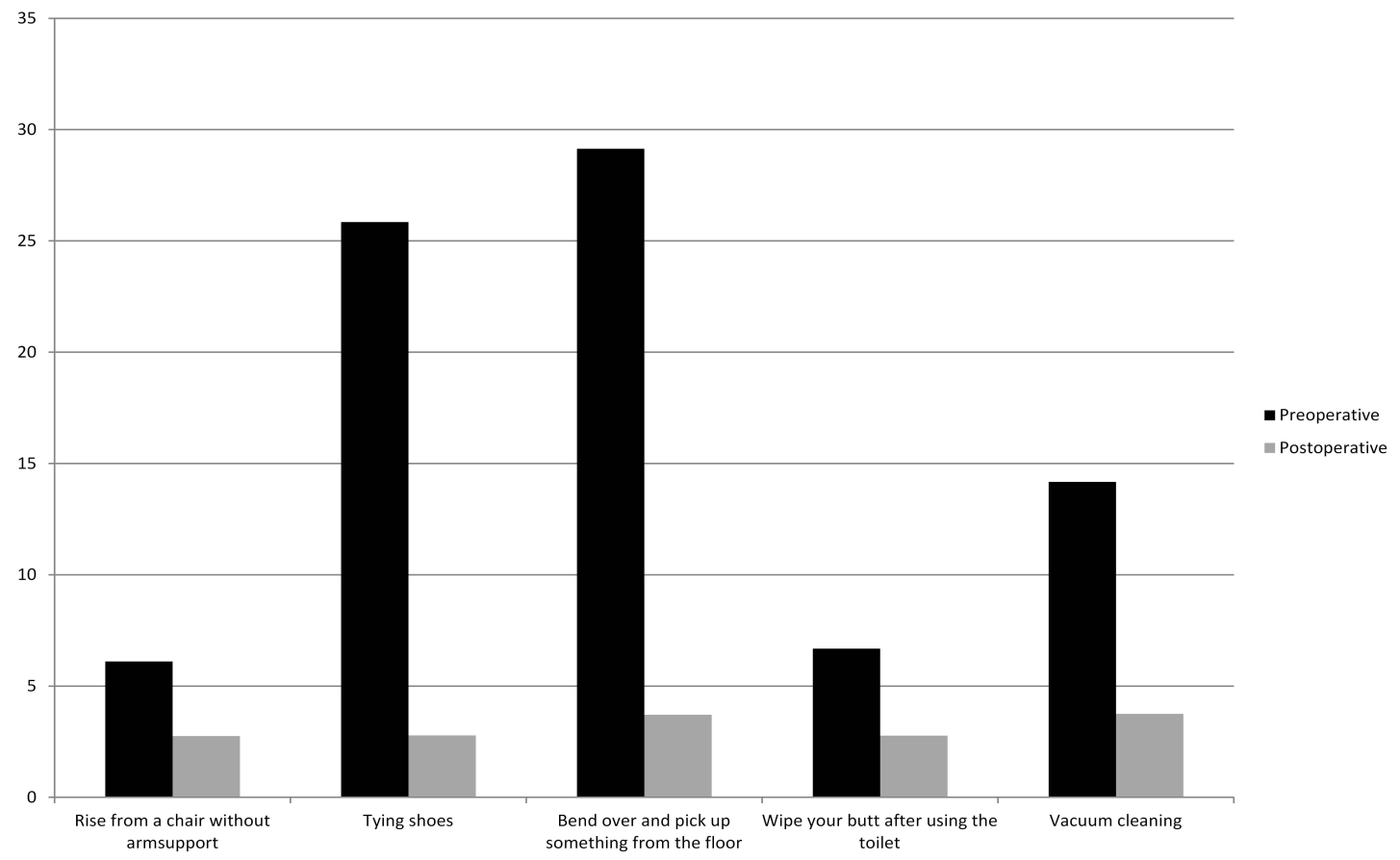

Fig. (2). The median of the five additional questions (in $\mathrm{mm}$ ) for the 70 patients preoperatively and 18 months postoperatively.

In this study, the median (min-max) EQ VAS score was 60 (20-100) $\mathrm{mm}$ preoperatively and 80 (20-100) $\mathrm{mm}$ postoperatively $(100=$ best imaginable health; $0=$ worst imaginable health). The difference between pre- and postoperative EQ VAS was significant $(p<0.001)$. Preoperatively, there was a significant difference between the EQ VAS scores and the Swedish reference values [8] $(\mathrm{p}<0.001)$, while there was no significant difference postoperatively $(\mathrm{p}=0.524)$.
The correlations between the EQ VAS score and the DRI Index were $r_{s}=0.61(p<0.001)$ preoperatively and $r_{s}=0.48$ $(\mathrm{p}<0.001)$ postoperatively.

Table 1 shows the percentages of the patients that classify their own health status in the five different dimensions, on the three levels of severity: no problems; moderate problems; severe problems.

In this study, the median (min-max) EQ-5D index was preoperatively $0.87(0.43-0.97)$ and postoperatively 0.93 
Table 1. The percentages $(\%)$ of the patients that classified their own health status in the five different dimensions, on the three levels of severity: no problems; moderate problems; severe problems.

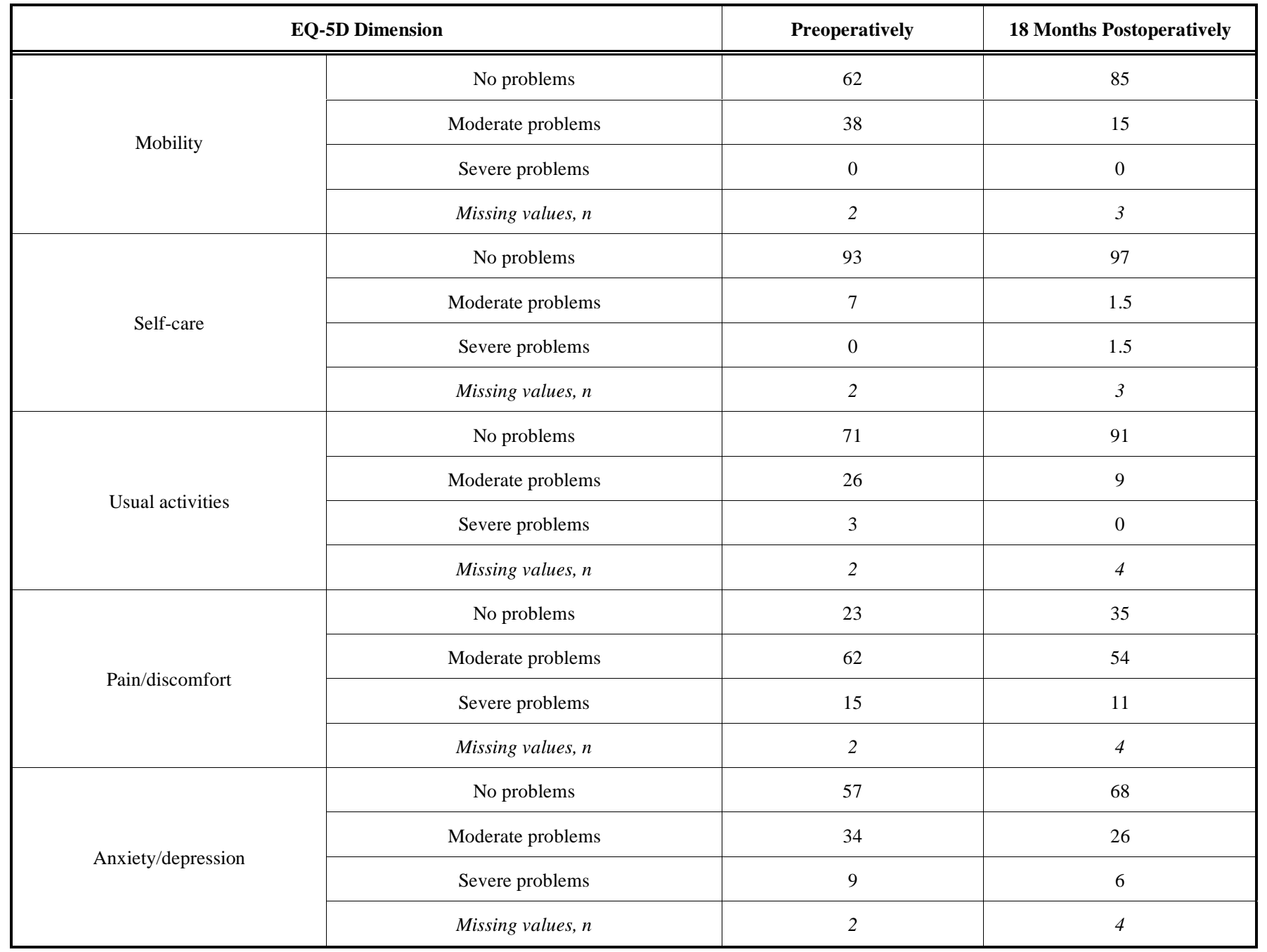

(0.43-0.97) $(1=$ the value for full health; $0=$ death $)$. The difference between the pre- and postoperative EQ-5D index was significant $(\mathrm{p}<0.001)$. The difference between the preoperative EQ-5D index and the Swedish reference values [8] was significantly lower $(\mathrm{p}<0.001)$ but not the postoperative EQ-5D index $(\mathrm{p}=0.919)$.

\section{DISCUSSION}

The results of physical function measured by Disability Rating Index from the patients participating in our trial was a total mean index score of $30.4 \mathrm{~mm}$ before surgery and 14.2 $\mathrm{mm} 18$ months after. The preoperative value corresponds to "Mild-to-moderate" disability according to Salén et al. [5] and are similar to physical function for people with pain in the neck, shoulders and low back as well as arthritis in knee and hip [5]. The postoperative value correspond to "Some" disability and found among healthy people presenting minor ailments [5]. It is well known that obesity causes limitation in physical function but this is the first trial where the DRI index has been used to describe physical function in an obese population.
The results of our study indicate that all activities included in DRI were experienced as being significantly less difficult to perform postoperatively than preoperatively, 18 months before. Even though the activities had become easier 18 months after the LRYGB, we cannot, on the basis of this study, say how important it is for the individuals. However, while the DRI questionnaire is self-administered and quick to complete, it is still a good alternative, in both the clinical setting and research, for collecting information about patients' disability by assessing activity and participation limitations before and after LRYGB. Further work is needed to establish definitive DRI anchor points identified as relevant or important to patients before and after bariatric surgery.

In addition to the 12 activities included in the DRI the patients also estimated their ability to perform five further activities that are clinically well-known to be troublesome for obese people. The five activities include ones in which it is necessary to bend forward, reach around oneself or rise. The questions have not yet been tested for validity or reliability but are based on clinical experience and evaluated in the same manner as the activities in DRI. This lack of a 
scientific basis is a limitation but there are, to our knowledge, no specific ADL or activity scores for people with obesity. As people with obesity have specific limitations and needs, there is a strong need to construct well-designed scores for ADL and physical function.

There were only low correlations between preoperative weight/BMI and the 12 activities or "total mean index" in DRI or the additional activities. The same pattern was seen 18 months postoperatively. A small sample size or large ranges in results of the tests included can explain the lack of correlations. This needs to be further evaluated in larger samples. One other explanation might be that the variations in body composition and fat distribution may impair the functional ability in various ways. Vincent et al. [10] conclude that a difference in body composition and fat allocation may impair lower body functional ability differently and that it is not clear which body composition is most closely associated with poor mobility. The results of two qualitative interview studies show that there are many obstacles that reduce patients' physical capacity both preoperatively [11] and one year after surgery [12]. One year after bariatric surgery, the patients still experienced their physical body as a hinder that prevented them from being physically active [12].

In a Swedish study that included over 40000 adult respondents, the mean EQ VAS was 79.5 millimeters [8]. The corresponding figures in our study were (in median (min-max)) 60 (20-100) mm preoperatively and 80 (20-100) $\mathrm{mm}$ postoperatively. The difference between our patients' preoperative EQ VAS scores and the Swedish reference values [8] was significantly lower $(\mathrm{p}<0.001)$, but no significant difference was seen postoperatively $(p=0.524)$. Several other studies have shown improved HRQoL after LRYGB, for example Kolotkin et al. [13] and Aftab et al. [14]. They showed improved and maintained HRQoL, measured by SF36, five to six years after LRYGB. Raoof et al. [15] also showed that, as long as seven to 17 years (average 12 years) after surgery, patients report better HRQoL, measured by SF36, compared to nonoperated obese controls; however, the patients in this study did not score as high as the general population.

In the present study, the median (min-max) EQ-5D index was preoperatively $0.87(0.43-0.97)$ and postoperatively 0.93 (0.43-0.97). These values correspond to fair health (values 0.8349 to 0.9442 ) based on Swedish reference values by Burström et al. [8]. The difference between the pre- and postoperative EQ-5D index and the Swedish reference values [8] was significantly lower $(\mathrm{p}<0.001)$ preoperatively but not postoperatively $(\mathrm{p}=0.919)$. The relatively low preoperative health related quality of life significantly increased 18 months postoperatively in this group of patients, but individuals still rate themselves as having very poor health (values <0.4959) [8] and this problem must be addressed by the healthcare system.

\section{CONCLUSION}

Both the HRQoL and the self-experienced ability to perform various daily physical activities increased significantly 18 months after LRYGB, compared to preoperative values.

\section{CONFLICT OF INTEREST}

The authors confirm that this article content has no conflict of interest.

\section{ACKNOWLEDGEMENTS}

This study was supported by grants from the Research and Development Council of Göteborg and Bohuslän.

\section{REFERENCES}

[1] Forhan M, Gill SV. Obesity, functional mobility and quality of life. Best Pract Res Clin Endocrinol Metab 2013; 27(2): 129-37.

[2] Sjöstrom L. Bariatric surgery and reduction in morbidity and mortality: experiences from the SOS study. Int J Obes 2008; 32: 93-7.

[3] Sjöström L, Narbro K, Sjöström CD, et al. Effects of bariatric surgery on mortality in Swedish obese subjects. N Engl J Med 2007; 357(8): 741-52.

[4] Ryden A, Torgerson JS. The swedish obese subjects study-what has been accomplished to date? Surg Obes Relat Dis 2006; 2(5): 549-60.

[5] Salen BA, Spangfort EV, Nygren AL, et al. The disability rating index: an instrument for the assessment of disability in clinical settings. J Clin Epidemiol 1994; 47(12): 1423-35.

[6] Parsons H, Bruce J, Achten J, et al. Measurement properties of the disability rating index in patients undergoing hip replacement. Rheumatology 2015; 54(1): 64-71.

[7] Rabin R, de Charro F. EQ-5D: a measure of health status from the EuroQol Group. Ann Med 2001; 33(5): 337-43.

[8] Burstrom K, Sun S, Gerdtham UG, et al. Swedish experience-based value sets for EQ-5D health states. Qual Life Res 2014; 23(2): 43142.

[9] Munro BH. Statistical methods for health and health care research. $5^{\text {th }}$ ed. Philadelphia: Lippincott Williams \& Wilkins 2005.

[10] Vincent HK, Vincent KR, Lamb KM. Obesity and mobility disability in the older adult. Obes Rev 2010; 11(8): 568-79.

[11] Wiklund M, Olsén MF, Willén C. Physical activity as viewed by adults with severe obesity, awaiting gastric bypass surgery. Physiother Res Int 2011; 16(3): 179-86.

[12] Wiklund M, Olsén MF, Olbers T, et al. Experiences of Physical activity one year after bariatric surgery. Open Obes J 2014; 36: 21 49.

[13] Kolotkin RL, Davidson LE, Crosby RD, et al. Six-year changes in health-related quality of life in gastric bypass patients versus obese comparison groups. Surg Obes Relat Dis 2012; 8(5): 625-33.

[14] Aftab H, Risstad H, Sovik TT, et al. Five-year outcome after gastric bypass for morbid obesity in a Norwegian cohort. Surg Obes Relat Dis 2014; 10(1): 71-8

[15] Raoof M, Naslund I, Rask E, et al. Health-related quality-of-life (hrqol) on an average of 12 years after gastric bypass surgery. Obes Surg 2015; [Epub ahead of print]. 